\title{
Trastorno Alimentario y su Relación con la Imagen Corporal y la Autoestima en Adolescentes
}

\author{
Eating Disorder and its Relationship with Body Image and Self-Esteem in Adolescents
}

\author{
Miriam Angélica Moreno González ${ }^{1}$ \\ Godeleva Rosa Ortiz Viveros ${ }^{2}$ \\ Universidad Veracruzana, México
}

(Rec: 19 de junio de 2009 - Acep: 27 de octubre de 2009)

\begin{abstract}
Resumen
Los trastornos en la conducta alimentaria constituyen un problema de salud por su creciente incidencia, gravedad de la sintomatología asociada, resistencia al tratamiento y porque se presenta en edades más tempranas. Se estudió la relación entre las conductas de riesgo de trastorno alimentario, la imagen corporal y autoestima en un grupo de adolescentes de Secundaria. Se aplicaron tres instrumentos: el Test de Actitudes Alimentarias (EAT-40), Cuestionario de la figura corporal (BSQ), Cuestionario de Evaluación de la Autoestima y un Cuestionario de datos sociodemográficos a 298 alumnos: 153 mujeres y 145 hombres, con una edad promedio de 13 años. Se encontró riesgo de trastorno alimentario (8\%) y en un $10 \%$ la patología instalada, con mayor prevalencia en las mujeres; ambos sexos presentan preocupación por su imagen corporal, niveles medios y bajos de autoestima y una mayor tendencia a presentar trastornos alimentarios en las mujeres. Los casos se incrementan con la edad.
\end{abstract}

Palabras claves: Trastorno alimentario, imagen corporal, autoestima.

\begin{abstract}
Eating disorders are now a public health problem, by its increasing incidence; severity of symptoms, its resistance to treatment and its appearance at an increasingly younger age to remain until adulthood. The study analyzes the relationship between risk-taking behaviors and eating disorder, body image and self-esteem in adolescents from secondary education. Instruments: Three instruments were applied: the eating Attitudes Test (EAT-40), the Body Shape Questionnaire (BSQ), the Assessment Questionnaire self-esteem, and a Sciodemographic Questionnaire. The sample were 298 students: 153 girls and 145 boys with an average of 13 years age. The results indicate that the risk of eating disorder is $8 \%$ and $10 \%$ with the disease installed; being more prevalent in women. Both sexes show dissatisfaction with their body image and low to medium self-esteem levels, with a higher trend in girls, and the cases are increasing with increasing age.
\end{abstract}

Key words: Eating disorders, body image, self-esteem.

1 Correspondencia a: Miriam Angélica Moreno González, Universidad Veracruzana, Instituto de Investigaciones Psicológicas. Av. Luis Castelazo Ayala S/N Col. Industrial Las Ánimas. E-mail: rene_angelica297@ hotmail.com. Tel. (22 8) 8418913 Ext. 13208

2 Godeleva Rosa Ortiz Viveros, Universidad Veracruzana, Instituto de Investigaciones Psicológicas. Av. Luis Castelazo Ayala S/N Col. Industrial Ánimas. . E- mail: godelevarosa@ hotmail.com roortiz@uv.mx.Tel. (22 8) 8418913 Ext.13205 


\section{Introducción}

Los trastornos de la conducta alimentaria constituyen hoy un problema de salud pública endémico, por su creciente incidencia, gravedad de la sintomatología asociada, su resistencia al tratamiento y porque se presenta en edades cada vez más tempranas, persistiendo hasta la edad adulta.

Los datos epidemiológicos indican que el ideal de delgadez es una grave amenaza y problema de salud, la anorexia y la bulimia conducen a la muerte en el $6 \%$ de los casos que lo padecen (Facts of life, 2002, citado en Tayler, 2007). En cuanto a la prevalencia, Schvili y Dayán (2000) señalan que ésta fluctúa entre el 0.5 y el $1 \%$ de acuerdo al DSM-IV (2002) para los cuadros clínicos clásicos de anorexia y bulimia, aunque existen hallazgos contradictorios en cuanto a cuál de ellos es el de mayor prevalencia. Barriguete, en el 2003, menciona que en las sociedades occidentales industrializadas estos trastornos presentan una mayor prevalencia y ocurren en todas las clases sociales, no sólo en niveles socioeconómicos medio y alto. Una revisión hecha por Anaya en el 2004, mostró que la tendencia de morbilidad en estudios referidos desde 1970 a 2002 , indicaban un $6 \%$ de incidencia y $17 \%$ de prevalencia, incluyendo aquellos que hacen referencia exclusivamente a la anorexia, los que sólo lo hacen a la bulimia y los que engloban y consideran todos los trastornos de la conducta alimentaria en su conjunto. Respecto a la edad de inicio de estos trastornos, se encontró que la edad promedio es de 17 años, aunque hay algunos datos que proponen edades entre 14 y 18 años. La Facts of life, 2002 (citada en Taylor, 2007) reporta que las más afectadas suelen ser las mujeres entre 15 y 24 años, pero se han detectado casos de trastorno alimentario en niños de apenas siete años y en personas de más de ochenta años. Por género, se ha visto un aumento de la incidencia de los trastornos alimentarios en las mujeres adolescentes de los países de occidente (Taylor, 2007). Por su parte, la National Eating Disorders Association (NEDA, 2002) considera que la bulimia se presenta entre el 1 y $2 \%$ de mujeres adolescentes y mujeres jóvenes de la población americana afectadas por estos trastornos. Quintero, Pérez, Montiel, Pirela, Acosta y Pineda, en el 2003, encontraron en la población venezolana, una mayor prevalencia de bulimia en mujeres $(0.82 \%)$ y una proporción menor en varones $(0.75 \%)$. La Encuesta Nacional de Epidemiología Psiquiátrica realizada en México, no reportó casos de anorexia, aunque informó sobre una prevalencia de bulimia en el 1.8\% en mujeres de 18 a 29 años de edad (Medina, Borges, Lara, Benjet, Blanco, Fleiz, et al. 2003).

En México, las investigaciones de los trastornos alimentarios donde se ha estudiado la valoración de la imagen corporal y la dieta restrictiva en niños y adolescentes se han realizado en población femenina, son escasos los que han incluido a los hombres (Álvarez, Franco, Vázquez, Escarria, Haro \& Mancilla 2003). Sin embargo, estudios realizados en distintos estados de la República Mexicana han demostrado que tanto los hombres como las mujeres presentan conductas de riesgo alimentario, como el realizado por Unikel, Saucedo, Villatoro y Fleiz (2002), donde mencionan que en los hombres, suele prevalecer una marcada tendencia a desear un cuerpo más corpulento y a mantener algunas conductas de riesgo como la práctica excesiva de ejercicio y comer compulsivamente. Asimismo, Cruz, Ávila, Cortés, Vásquez y Mancilla (2008), en un estudio realizado con adolescentes universitarios mexicanos, encontraron que los hombres manifiestan mayor sintomatología de trastornos alimentarios, insatisfacción corporal y conductas de riesgo alimentario que las mujeres. Caso contrario fue hallado por Aguilera y Milián (2006) en escuelas privadas de otro estado diferente, pero en el propio país, en el que las mujeres de entre 18 y 19 años de edad, presentaban un mayor riesgo de padecer algún trastorno alimentario. Por su parte, Vázquez, Mancilla, Mateo, López, Álvarez y Ruiz (2005), reportaron que los hombres realizan ejercicio físico excesivo, mientras que las mujeres realizan dietas restrictivas rígidas o ayunan. Hernández y Laviada (2005) encontraron en estudiantes de preparatoria, una frecuencia alta de conductas de riesgo alimentario, como vómito autoinducido, ayuno, sobrevaloración de la importancia del peso y la figura corporal como satisfactores personales y dieta restrictiva. Del Bosque y Ehrenzweig (2004) realizaron un Taller para la prevención de trastornos alimentarios. El taller "El espejo de mî", en una escuela secundaria pública, resultó un instrumento útil para mejorar la imagen corporal de las jóvenes y elevar ciertas dimensiones de la autoestima y del autoconcepto, pero no así para el autocontrol. Juárez y Ehrenzweig (2006) reportan que en estudiantes de una Preparatoria privada, un sexto de la población estudiada presenta riesgo de trastorno alimentario. Encontraron correlaciones positivas con los factores de restricción y desinhibición, pues aunque evitan comer alimentos altos en calorías, cuando los ingerían se sentían culpables. Encontraron, además, que las mujeres de la muestra estaban más preocupadas por su cuerpo, deseaban estar más delgadas y realizaban más ejercicio que los hombres, con la finalidad de quemar calorías y así disminuir su peso corporal. En cuanto a la edad de inicio de los trastornos alimentarios, Unikel (2000) menciona que las edades de inicio más frecuentes son entre 18 y 19 años. Sin embargo, en otro estudio realizado por Unikel, Saucedo, Villatoro y Fleiz (2002), los resultados muestran una edad de inicio de 16.5 años. Como dato adicional, Vázquez, Mancilla, Mateo et al. (2005), reportaron un porcentaje elevado de conductas compensatorias no purgativas, como inducirse al vómito o hacer ejercicios de manera extenuante para estar delgados, en jóvenes de 15 años, es decir, una edad menor a la encontrada anteriormente en jóvenes mexicanas; conforme pasa el tiempo se reduce cada vez la edad en que ocurren los trastornos alimentarios.

En el tema de los trastornos alimentarios, una de las variables estudiadas es la de la imagen corporal que perciben 
las personas, ésta se ha asociado frecuentemente con la modificación nociva de los hábitos alimentarios, hasta llegar a constituir un problema de salud. Se ha sugerido que la alteración de la percepción de la imagen corporal es un síntoma precoz de la presencia de trastornos alimentarios como anorexia y bulimia (Sánchez, 2001). Baile, en el 2002, agrega que "una alteración de la imagen corporal ó insatisfacción corporal, se ha considerado clave dentro de los posibles factores predisponentes a las distorsiones en la percepción del tamaño corporal, como un criterio diagnóstico, y ha provocado que se estudie como una variable asociada a los trastornos de la conducta alimentaria". Se han encontrado en la literatura, otras definiciones sobre la distorsión preceptual de la imagen corporal, como la expresada por Sepúlveda, Botella y León (2001), quienes la describen como "la presencia de juicios valorativos sobre el cuerpo que no coinciden con las características reales". Sin embargo, no existe un consenso en la definición de la imagen corporal y resulta necesario especificar, en cada estudio, el aspecto de la imagen corporal que se considera alterado. En este caso, el término "distorsión perceptual" se entiende como la alteración de la imagen corporal en lo relativo a la estimación de tamaño e "insatisfacción corporal" como el conjunto de emociones, pensamientos y actitudes negativos hacia el tamaño y la forma del cuerpo.

Para Chávez (2004) la imagen corporal representa una construcción social con referentes biológicos, psicológicos, económicos y socioculturales, por lo que espacios culturales diversos dan lugar a patrones estético corporales específicos para cada uno.

Por su parte, los medios de comunicación influyen en la existencia de un modelo ideal de belleza establecido y compartido socialmente, lo que se traduce en una presión significativa para los miembros de una sociedad, por la importancia que se concede al atractivo para triunfar en todas las áreas. Unikel (2000) menciona que la insatisfacción corporal, la excesiva preocupación por el peso y los trastornos alimentarios han venido incrementándose de manera consistente en las escuelas. Se han realizado investigaciones desde la perspectiva de las teorías del desarrollo en las que se hace hincapié en el papel que desempeña el desarrollo de la infancia y de la adolescencia en la distorsión de la imagen corporal. Unikel, Saucedo, Villatoro y Fleiz (2002) argumentan que en algunos estudios se ha demostrado que entre más afectado emocionalmente se encuentre un adolescente, menos soportará su apariencia física y por ende será más susceptible a desarrollar trastornos alimentarios.

Montero, Morales y Carbajal, en el 2004, demostraron que la imagen corporal es un buen predictor de los trastornos alimentarios y que los adolescentes tienden a despreciar un cuerpo con sobrepeso, debido a que socialmente existe la creencia de que un cuerpo delgado es el ideal; Asimismo, mencionan que el interés por estudiar las alteraciones en la percepción de la imagen corporal es cada vez mayor, en los estudios realizados se ha detectado que múltiples factores biológicos, sociales y culturales modulan esta percepción, sin embargo, todavía no se han desarrollado suficientes métodos objetivos para su valoración y en algunos casos estos factores son difíciles de estimar (Gupta, 2000). Al respecto, Dowson y Hendersen (2001) comentan que la percepción de la imagen corporal se utiliza a menudo como medida de la autoestima y del grado de satisfacción con la propia imagen.

Abundando un tanto en el tema de la autoestima, Aramburu y Guerra, 2001 la definen como "una especie de sociómetro que indica el grado en que una persona se percibe como incluida o excluida en el entramado social", de modo que una de las principales funciones que se atribuyen a la autoestima es la protección de la exclusión social, esto es, la manera en que las personas tratan de manejar la impresión que causan a los demás, con estrategias que aseguren su integración social y eviten su exclusión. Para García (1998) la autoestima se refiere a la forma en que las personas se perciben a sí mismas en términos generales, independientemente del aspecto analizado.

Martínez, en el 2001, menciona que científicos del desarrollo humano, como Peretz, Elkins, Rogers, Maslow, Bettleheim, afirman que la autoestima es una parte fundamental para que el hombre alcance su plenitud y plena expresión de sí mismo. La persona, en su autoevaluación, puede verse con orgullo o vergüenza, considerar que posee valor o falta de él. Según algunos estudios citados por Ortuño (1978), las personas que presentan una autoestima alta, muestran un control de sus impulsos mayor, con más frecuencia se auto refuerzan, refuerzan a otros más a menudo, se auto afirman, son persistentes en la búsqueda de soluciones constructivas, poco agresivos y muestran una actitud positiva hacia su propio cuerpo. Otros autores como Toro (1996) y Rosen y Ramírez (1998) destacan que la baja autoestima es uno de los principales síntomas presentes en las personas diagnosticadas con trastornos de la alimentación, expresándose con inseguridad, falta de aprobación y de respeto hacia sí mismas. La preocupación acerca del peso y la insatisfacción por la imagen corporal constituyen los dos elementos básicos sobre los que se asienta esta baja autoestima como producto de inferencias y creencias, muchas de ellas sujetas a distorsiones.

El papel que juegan las variables (magen corporal, autoestima y diversos factores sociodemográficos relacionados con los trastornos alimentarios) se estudió en esta investigación.

\section{Método}

\section{Sujetos}

La muestra estuvo conformada por 298 adolescentes, alumnos de una escuela secundaria pública de la ciudad 
de Xalapa, Veracruz, México, de los cuales 51\% (153) eran mujeres y 49\% (145) hombres, con una edad de 13 a 14 años.

\section{Instrumentos}

Se elaboró un Cuestionario de datos generales para contextualizar a la población, y se registró la talla y peso actual, para obtener el índice de masa corporal (IMC), el cual consiste en la razón entre el peso expresado en kilos y la talla expresada en metros al cuadrado. Se utilizó además el Test de Actitudes Alimentarias (EAT) de Garner y Garfinkel (1979), este instrumento evalúa las características anoréxicas: Miedo a engordar, motivación para adelgazar y patrones alimentarios restrictivos. Consta de 40 ítems que se contestan en una escala de frecuencia de 6 puntos que va de "nunca" a "siempre", el punto de corte utilizado es el sugerido por el Ministerio de Sanidad y Consumo del Servicio Aragonés de salud (2000) en su Protocolo de Atención a pacientes con TCA, quienes proponen la siguiente clasificación: Normal $\leq 19$, riesgo 20-30 y caso clínico $\geq 31$. Otra escala utilizada fue el Cuestionario de la Figura corporal (BSQ) de Cooper (1987), el cual consta de 34 ítems que evalúan la insatisfacción corporal y la preocupación por el peso. Espina (2001) clasifica las puntuaciones en 4 categorías, para determinar los niveles de insatisfacción con la imagen corporal y preocupación por el peso en: No preocupado $\leq 80$, ligeramente preocupado $81-110$, moderadamente preocupado 111-140, extremadamente preocupado $\geq 140$. Y finalmente, el Cuestionario de Evaluación de la Autoestima para Alumnos de Enseñanza Secundaria (CEA) de García (1998), consta de 19 ítems, 16 de los cuales se refieren a cualidades propias de la autoestima y 3 pertenecen a una escala de autocrítica que trata de evaluar en qué medida los alumnos están contestando o no bajo los efectos de la deseabilidad social. Para obtener el nivel alto, medio o bajo de su autoestima, se realizó una estimación percentilar, en donde los puntajes obtenidos se dividieron en tres partes iguales de $33.33 \%$ cada una, considerando como población en riesgo la que alcanzó puntajes de 36-47 correspondientes al segundo tercil y como población afectada aquella cuyo puntaje era igual o mayor de 48 .

\section{Procedimiento}

Después de la selección de instrumentos se realizó un piloteo con 20 alumnos para conocer sobre su comprensibilidad y se realizó una validación de constructo a partir de la consulta a cinco expertos. Se capacitó y entrenó a los encuestadores en el llenado de los cuestionarios y en la toma de medidas antropométricas (peso, talla), utilizando una báscula calibrada, con taras de $0.1 \mathrm{~kg}$. La lectura se registró en kilos y gramos, bajo los siguientes criterios: El peso corporal se establece con la persona descalza, de pie sobre la parte central de la plataforma de la báscula y en forma simétrica. Para el registro de la talla se usó un flexómetro y una escuadra, considerando que la talla de pie es referida como la distancia máxima comprendida entre el suelo y el vértex (punto más alto de la cabeza), el sujeto permanece sin zapatos, completamente erguido, con los talones juntos, los brazos por un lado del cuerpo y de espaldas a la pared sobre una superficie plana a la cual se fijó el flexómetro, el registro se reportó en centímetros y milímetros (González, 2006). Con base en los listados de asistencia se seleccionó la muestra aleatoriamente. Se aplicaron los cuestionarios en los salones de clases y las medidas antropométricas en un espacio apropiado. El análisis de los datos se llevó a cabo mediante el paquete estadístico Statistica versión 6. Se analizó el comportamiento de las variables de estudio mediante estadística descriptiva, y para determinar la correlación entre las variables se utilizó el coeficiente de correlación de Pearson.

\section{Resultados}

La población quedó integrada por 298 jóvenes entre los 12 y 14 años de edad. La medición del índice de masa corporal permitió detectar 33 casos de desnutrición (13 en hombres y 20 en mujeres) y 41 casos de obesidad de tipo I y II en los hombres y 37 mujeres con obesidad, en sus tres niveles de clasificación, como se muestra en la Tabla 1.

Se detectó riesgo de trastorno alimentario en el $4 \%$ de los hombres y el $12 \%$ de las mujeres y la patología ya instalada en un $10 \%$ en ambos sexos, como se muestra en la Tabla 2.

Como se aprecia en la Figura 1, la edad en la que se presentan el riesgo y los casos clínicos es a los 14 años, con una mayor prevalencia en las mujeres.

La preocupación con su imagen corporal muestra una tendencia mayor en las mujeres, pero también se presenta en los hombres. Como se puede apreciar en la Tabla 3.

Al observar la Tabla 4 se aprecia que los hombres presentan preocupación con su imagen corporal en los tres grupos de edad, aun cuando ésta tiende a bajar al incrementarse la edad, y ocurre en sólo dos casos que equivalen a un $1 \%$ de la población varonil en la edad de 13 años, en la categoría extremadamente preocupados.

Como se aprecia en la Tabla 5 la preocupación con su imagen corporal, en las mujeres se observa un incremento conforme avanza su edad, lo que nos indica, como en el caso de los hombres, que ya hay una patología instalada en estas jovencitas.

En cuanto a su autoestima, los casos se concentran más en el nivel medio en ambos sexos y ambos coinciden en un $10 \%$ de los mismos, en los niveles bajos (Tabla 6).

Por grupo de edad hay un incremento de los casos en el nivel medio de autoestima en los hombres a la edad de 
Tabla 2. Distribución de la muestra por nivel de riesgo de trastorno alimentario según sexo

\begin{tabular}{|c|c|c|c|c|c|c|}
\hline \multirow[b]{2}{*}{ EAT 40} & \multicolumn{2}{|c|}{ Hombres } & \multicolumn{2}{|c|}{ Mujeres } & \multicolumn{2}{|c|}{ Subtotal } \\
\hline & $\mathrm{fr}$ & $\%$ & fr & $\%$ & fr & $\%$ \\
\hline Normal & 125 & 86 & 119 & 78 & 244 & 82 \\
\hline Riesgo & 5 & 4 & 19 & 12 & 24 & 8 \\
\hline Caso clínico & 15 & 10 & 15 & 10 & 30 & 10 \\
\hline Total & 145 & 100 & 153 & 100 & 298 & 100 \\
\hline
\end{tabular}

Fuente: Investigación sobre riesgo alimentario, imagen corporal y autoestima 2006

Figura 1. Distribución por sexo de la población en riesgo de trastorno alimentario según edad

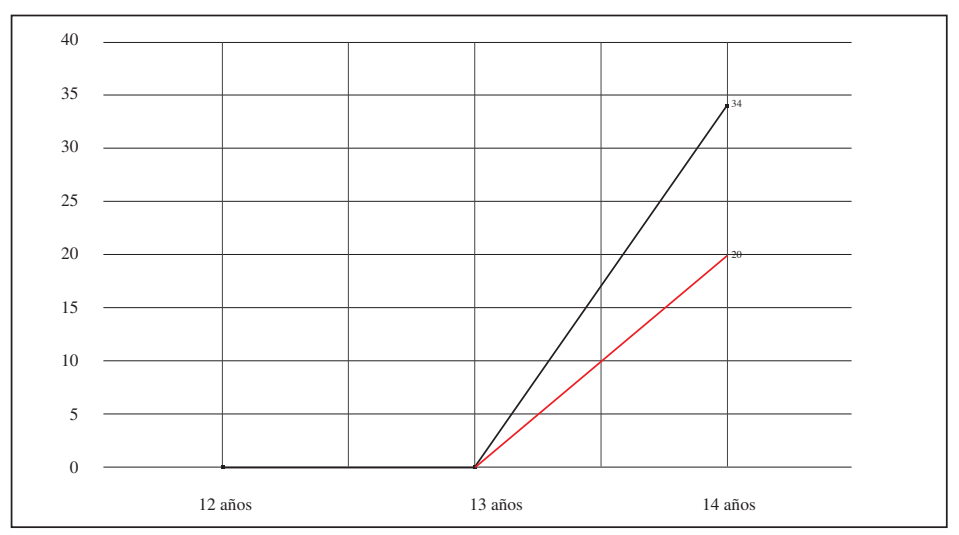

Tabla 3. Distribución de la población de la muestra por nivel de preocupación con su imagen corporal, según sexo

\begin{tabular}{|c|c|c|c|c|c|c|}
\hline \multirow{2}{*}{ BSQ } & \multicolumn{2}{|c|}{ Hombres } & \multicolumn{2}{|c|}{ Mujeres } & \multicolumn{2}{|c|}{ Subtotal } \\
\hline & $\mathrm{Fr}$ & $\%$ & $\mathrm{fr}$ & $\%$ & $\mathrm{fr}$ & $\%$ \\
\hline No preocupado & 127 & 88 & 101 & 66 & 228 & 77 \\
\hline Ligeramente preocupado & 10 & 7 & 26 & 17 & 36 & 12 \\
\hline Moderadamente preocupado & 6 & 4 & 12 & 8 & 18 & 6 \\
\hline Extremadamente preocupado & 2 & 1 & 14 & 9 & 16 & 5 \\
\hline Total & 145 & 100 & 153 & 100 & 298 & 100 \\
\hline
\end{tabular}

Fuente: Investigación sobre riesgo alimentario, imagen corporal y autoestima 2006

$13(45 \%)$ a 14 años (52\%) y en el nivel bajo se presenta en los 13 años, caso contrario en las mujeres pues dicho incremento se presenta a la edad de 12 años (cuadro 7).

En la Tabla 8 se concentran las correlaciones entre las variables habiendo elegido un nivel $\mathrm{p} \leq .05000$, para considerar los datos como significativos. La lectura de la información nos permite afirmar que la correlación entre la conducta de riesgo de trastorno alimentario y la imagen corporal, muestra una relación positiva, lo que indica que a mayor puntaje en trastorno alimentario, mayor insatisfacción con su imagen corporal, dato que confirma la hipótesis de investigación: Las conductas de riesgo de trastorno alimentario guardan una relación directa con la insatisfacción de la imagen corporal en los adolescentes de la Escuela Secundaria General núm. 1 Lic. Sebastián Lerdo de Tejada.

En lo relativo a la relación entre trastornos alimentarios y la autoestima los resultados son contundentes, indicando una relación significativa negativa entre ambas variables, es decir, que al aumentar los puntajes en trastornos alimentarios, los puntajes en autoestima se decrementan. Sin embargo, en contra de lo esperado, el índice de masa corporal no muestra una relación estadísticamente significativa $\leq$ a 0.05 con los puntajes en trastornos alimentarios, aun cuando los datos indican una relación con tendencia positiva entre ellas, esto es, a mayor conducta de riesgo de trastorno alimentario, mayor índice de masa corporal. En cuanto a la correlación entre los trastornos alimentarios y la 
Tabla 4. Distribución de la muestra de hombres con preocupación con su imagen corporal, según edad

\begin{tabular}{|c|c|c|c|c|c|c|c|c|c|}
\hline \multirow{3}{*}{ Niveles BSQ } & \multirow{3}{*}{ Edad } & \multicolumn{8}{|c|}{ Hombres } \\
\hline & & \multicolumn{2}{|c|}{12} & \multicolumn{2}{|c|}{13} & \multicolumn{2}{|c|}{14} & \multicolumn{2}{|c|}{ Subtotal } \\
\hline & & fr & $\%$ & fr & $\%$ & fr & $\%$ & fr & $\%$ \\
\hline No preocupado & & 46 & 32 & 40 & 28 & 41 & 28 & 127 & 88 \\
\hline Ligeramente preocupado & & 4 & 3 & 3 & 2 & 3 & 2 & 10 & 7 \\
\hline Moderadamente preocupado & & 2 & 1 & 2 & 1 & 2 & 1 & 6 & 4 \\
\hline Extremadamente preocupado & & 0 & 0 & 2 & 1 & 0 & 0 & 2 & 1 \\
\hline Total & & 52 & 36 & 47 & 32 & 46 & 31 & 145 & 100 \\
\hline
\end{tabular}

Fuente: Investigación sobre riesgo alimentario, imagen corporal y autoestima 2006

Tabla 5. Distribución de la muestra de mujeres con preocupación con su imagen corporal, según edad

\begin{tabular}{|c|c|c|c|c|c|c|c|c|}
\hline \multirow{3}{*}{$\begin{array}{l}\text { Edad } \\
\text { Niveles BSQ }\end{array}$} & \multicolumn{8}{|c|}{ Mujeres } \\
\hline & \multicolumn{2}{|c|}{12} & \multicolumn{2}{|c|}{13} & \multicolumn{2}{|c|}{14} & \multicolumn{2}{|c|}{ Subtotal } \\
\hline & $\mathrm{fr}$ & $\%$ & $\mathrm{fr}$ & $\%$ & $\mathrm{fr}$ & $\%$ & $\mathrm{fr}$ & $\%$ \\
\hline No preocupado & 34 & 22 & 34 & 22 & 33 & 22 & 101 & 66 \\
\hline Ligeramente Preocupado & 5 & 3 & 9 & 6 & 12 & 8 & 26 & 17 \\
\hline Moderadamente preocupado & 2 & 1 & 2 & 1 & 8 & 5 & 12 & 8 \\
\hline Extremadamente preocupado & 2 & 1 & 4 & 3 & 8 & 5 & 14 & 9 \\
\hline Total & 43 & 27 & 49 & 32 & 61 & 40 & 153 & 100 \\
\hline
\end{tabular}

Fuente: Investigación sobre riesgo alimentario, imagen corporal y autoestima 2006

Tabla 6. Distribución de la muestra por nivel de autoestima según sexo

\begin{tabular}{|c|c|c|c|c|c|c|}
\hline \multirow[b]{2}{*}{ Nivel } & \multicolumn{2}{|c|}{ Hombres } & \multicolumn{2}{|c|}{ Mujeres } & \multicolumn{2}{|c|}{ Subtotal } \\
\hline & $\mathrm{Fr}$ & $\%$ & fr & $\%$ & fr & $\%$ \\
\hline Alto & 62 & 43 & 60 & 39 & 122 & 41 \\
\hline Medio & 68 & 47 & 78 & 51 & 146 & 49 \\
\hline Bajo & 15 & 10 & 15 & 10 & 30 & 10 \\
\hline Total & 145 & 100 & 153 & 100 & 298 & 100 \\
\hline
\end{tabular}

Fuente: Investigación sobre riesgo alimentario, imagen corporal y autoestima 2006

Tabla 7. Distribución de la muestra por sexo según nivel de autoestima y edad

\begin{tabular}{|c|c|c|c|c|c|c|c|}
\hline \multirow[b]{2}{*}{ Edad } & \multirow{3}{*}{ Sexo } & \multicolumn{6}{|c|}{ Niveles de autoestima } \\
\hline & & \multicolumn{2}{|c|}{ Alto } & \multicolumn{2}{|c|}{ Medio } & \multicolumn{2}{|c|}{ Bajo } \\
\hline & & $\mathrm{fr}$ & $\%$ & $\mathrm{fr}$ & $\%$ & $\mathrm{fr}$ & $\%$ \\
\hline \multirow{2}{*}{12} & $\mathrm{H}$ & 24 & 46 & 23 & 44 & 5 & 10 \\
\hline & M & 17 & 39 & 21 & 49 & 5 & 12 \\
\hline \multirow{2}{*}{13} & $\mathrm{H}$ & 20 & 43 & 21 & 45 & 6 & 12 \\
\hline & $\mathrm{M}$ & 18 & 37 & 27 & 55 & 4 & 8 \\
\hline \multirow{2}{*}{14} & $\mathrm{H}$ & 18 & 39 & 24 & 52 & 4 & 9 \\
\hline & M & 25 & 41 & 30 & 49 & 6 & 10 \\
\hline
\end{tabular}

Fuente: Investigación sobre riesgo alimentario, imagen corporal y autoestima 2006 
Tabla 8. Matriz de correlación de Pearson

\begin{tabular}{lcccc}
\hline $\mathrm{N}=298$ & & $\mathrm{r}(\mathrm{X}, \mathrm{Y})$ & $\mathrm{r}$ & $\mathrm{p}$ \\
\hline & BSQ & 0.711836 & 0.506711 & $* 0.000000$ \\
EATGLOBAL & AUTOESTIMA & -0.285691 & 0.081619 & $* 0.000001$ \\
& IMC & .101516 & .010305 & 0.080188 \\
& EDAD & .038243 & .001463 & 0.510763 \\
BSQ & AUTOESTIMA & -0.441874 & 0.195252 & $* 0.000000$ \\
& IMC & .295891 & .087552 & $* 0.000000$ \\
Autoestima & EDAD & .161733 & .026157 & $* 0.005132$ \\
IMC & IMC & -.093515 & .008745 & 0.107166 \\
*p & EDAD & -.044427 & .001974 & .027757 \\
\end{tabular}

$* \mathrm{p} \leq .05000$

Fuente: Investigación sobre riesgo alimentario, imagen corporal y autoestima 2006

variable edad, éstas no presentan una relación significativa, ni las tendencias esperadas.

La imagen corporal y la autoestima muestran una relación significativa negativa, lo que indica que a mayor preocupación con la imagen corporal, menor autoestima. En lo relativo al índice de masa corporal (IMC) la relación es significativa, es decir, a mayor índice de masa corporal, mayor preocupación con su imagen corporal. Por lo que respecta a la edad y su relación con la preocupación corporal, los resultados indican una relación estadísticamente significativa que indica que, conforme incrementa la edad, aumenta la preocupación con su imagen corporal. La misma variable edad, en relación con el índice de masa corporal, indica una relación significativa que se interpreta como: a mayor edad, menor índice de masa corporal.

\section{Discusión}

Al evaluar la relación entre los trastornos alimentarios, la imagen corporal y la autoestima de los adolescentes, se comprobó que las mujeres tienen mayor tendencia hacia este tipo de trastornos que los hombres, no obstante éstos también presentan el riesgo. Tanto hombres como mujeres se encuentran en una etapa de crecimiento y desarrollo en el que están constantemente evaluándose, presentan baja autoestima y se encuentran insatisfechos con su imagen corporal, lo que los puede llevar a realizar conductas para bajar de peso.

Al comparar por sexo, se observa que tanto los hombres como las mujeres presentan casos clínicos (10\%) y en nivel de riesgo se encuentran un $12 \%$ las mujeres vs. un $4 \%$ de los hombres, lo que denota una mayor proporción de casos clínicos con las mujeres. Dato similar fue encontrado por Chávez (2007) en población mexicana, donde las actitudes y conductas alimentarias se explicaron en un $18 \%$, debido al sexo, las mujeres obtuvieron mayor alteración de actitudes y conductas alimentarias que los hombres. Ruíz, Vázquez, Mateo, Galdós, Álvarez y Mancilla (2004) reportan también datos similares en cuanto a la sintomatología de Anorexia evaluada por el cuestionario EAT 40 en población mexicana, encontrándose mayor sintomatología de bulimia en hombres como en mujeres. La edad en que se presenta el riesgo de trastorno alimentario y caso clínico en esta investigación es a los 14 años, los hallazgos en investigación mencionan que los trastornos alimentarios y la insatisfacción con su imagen corporal se inician en las niñas alrededor de los 10 años y se incrementan con la transición de la escuela secundaria (Unikel, 2000).

En los datos encontrados en esta investigación, relativos a la insatisfacción con la imagen corporal, es posible observar que las mujeres presentan mayor preocupación por engordar y mayores porcentajes de riesgo de los trastornos alimentarios, quizá debido a que la presión social es más fuerte hacia ellas. Sin embargo, el grupo de los hombres presenta valores que denotan riesgo y en consecuencia, es imprescindible llevar a cabo programas preventivos de trastornos alimentarios que impidan el incremento del problema. Aun cuando en México la información sobre la presencia de trastornos alimentarios es insuficiente, al estudiar a mujeres mexicanas se ha encontrado que aquellas que presentan sintomatología de trastorno alimentario tienen mayor insatisfacción con la imagen corporal, más temor a engordar, así como sentimientos de baja autoestima ligada a la valoración de su cuerpo. Las investigaciones realizadas en distintos estados de la República Mexicana, dan cuenta de que los trastornos alimentarios ya se encuentran en instalación en la población de jóvenes estudiantes, en los niveles de educación media y superior tanto en escuelas públicas como privadas. Si bien los resultados de esta investigación no se pueden generalizar a toda la población, se muestran como antecedentes para futuras investigaciones.

Se requieren más estudios que ayuden a comprender estos trastornos, sin embargo, reconocer que los grupos 
estudiantiles son una población de alto riesgo para desarrollar trastornos alimentarios y que dichos trastornos se encuentran estrechamente relacionados con la preocupación por su imagen corporal y baja autoestima, son elementos clave para el diseño de programas preventivos de trastornos alimentarios y de tamizaje de casos para su tratamiento oportuno.

\section{Referencias}

Aguilera B. Ma. A \& Milián S. F. (2006). Mapa nutricio y riesgo de trastornos de la conducta alimentaria en adolescentes de la ciudad de Querétaro, México. Salud pública y Nutrición, 7(1) RESPYN [En línea] Consultado el 7/2/07 http://www.respyn.uanl.mx/vii/1/articulos/

Álvarez, R, G., Franco, P, K., Vázquez, A. R., Escarria, R, A. R., Haro, H. M. \& Mancilla, D, J. M (2003). Trastorno alimentario: Comparación entre hombres y mujeres estudiantes. Psicología y salud, 13(2), 246-247.

Anaya, V. (2004) El sexo, factor relevante en los trastornos de la conducta alimentaria. Revista de Enfermería Clínica, 14(4), 230-234.

Aramburu, M. \& Guerra, J. (2001). Autoconcepto: dimensiones, origen, funciones, incongruencias, cambios y consistencia. Interpsiquis, 2, 246-249.

Asociación Psiquiátrica Americana (APA, 2002). Manual Diagnóstico y Estadístico de los Trastornos mentales. (DSM-IV). Cuarta edición. Washington, DC.

Baile, A. J. I. (2002) ¿Qué es la imagen corporal? Centro Asociado de la UNED de Tudela [En línea] http://www.uned.es/ca-tudela/revista/ n002/baile_ayensa.htm

Barriguete, M. J. A. (2003). Anorexia y Bulimia Nerviosa: El Control Del Miedo. Cuadernos de Nutrición, 26(6), 280-289. Instituto Nacional de Ciencias Médicas y Nutrición Salvador Zubirán.

Cooper, (1987). Cuestionario de la Figura corporal (BSQ). En: Gutiérrez, T., Raich, R., Sanchez, D. \& Deus, J. (2003). Instrumentos de evaluación en trastornos del comportamiento alimentario y obesidad. Madrid: Ed. Alianza. Pp. 242, 257-260, 267-271

Cruz, B. R. M., Ávila E. L. M., Cortés S. M. C., Vásquez A. R. \& Mancilla, D. J. M. (2008). Restricción alimentaria y conductas de riesgo de trastornos alimentarios en estudiantes de Nutrición. Psicología y Salud, 18(002), 189-198

Chávez, H. A. M., Macías, G. L. F., Gutiérrez, M. R., Martínez, H. C. \& Ojeda, R. D (2004) Trastornos alimentarios en jóvenes guanajuatenses. Acta Universitaria, 14(002), 17-24.

Chávez , H. A. E. (2007). Actitudes y conductas alimentarias en adolescentes y su relación con índice de masa corporal. Salud comunitaria. Universidad Autónoma de Nuevo León, Facultad de Enfermería. Red de enfermería de América Latina. [ En línea] http://real.enfermeria uanl.mx

Del Bosque, Ch. Ma. L. \& Ehrenzweig, S. Y. (2004). El espejo de mí: Taller para la prevención de trastornos alimentarios. Tesis de licenciatura. Facultad de Psicología, Universidad Veracruzana. México.

Dowson, J. \& Hendersen, L. (2001). En: Montero, P., Morales, E. M. \& Carbajal A. (2004) Valoración de la percepción de la imagen corporal mediante modelos anatómicos. Antropología, 8, 107-116. www.didac. ehu.es/antropo

Facts of life. (2002). Conductas que mejoran la salud. En: Taylor S. E (2007). Psicología de la Salud. Trad. Hano R. M. C. \& Sánchez P. G. Pp. 16-39. Sexta edición. McGraw-Hill, México.

García G., A. (1998). Programa para la mejora de las habilidades sociales y la autoestima. Premios Joaquín Sama a la Innovación Educativa. Junta de Extremadura. [En Línea] consultado el 27/3/06 http://roble. cnice.mecd.es/ agarci19/Orientainterviene/Cuestautoestima/secundaria.htm

Espina, A., M. A. (2001). La imagen corporal en los trastornos alimentarios. Psicothema, 13(4), 533-538
Gardner, G. \& Garfinkel, S. (1979). Test de Actitudes Alimentarias (EAT) En: Gutiérrez, T., Raich, R., Sánchez, D. \& Deus, J. (2003) Instrumentos de evaluación en trastornos del comportamiento alimentario y obesidad. Madrid : Ed. Alianza. Pp. 242, 257-260, 267-271

Gupta, M. A., Chaturvedi, S. K., Chandarana, P. C. \& Johnson, A. M. (2000). Weight-related body image concerns among 18-24-years-old women in Canada and India. An empirical comparative study. Journal of Psychosomatic Research, 50, 193-198.

González, G. R. (2006). Estado nutricional y antropometría. Boletín de Educación Permanente en Salud. Material para la actualización médica, 1(1). Subdirección de Salud, Tlalpan, http://www.tlalpan.gob. $\mathrm{mx} /$ centros/otros/salud/eps1.pdf

Halmi, K. Eating disorders. En: Sadock, C. (2000). Comprehensive Textbook of Psychiatry. Philadelphia: Lippincott Williams and Wilkins. Pp. 1663-5

Hernández, E. V. \& Laviada, M. H. (2005). Frecuencia de bulimia nerviosa y conductas alimentarias de riesgo en estudiantes de preparatorias públicas y privadas de Mérida, Yucatán. Salud Pública y Nutrición,16, RESPYN [En línea] Consultado el 7/2/07 http://www.uanl.mx/publicaciones/respyn/especiales/2005/ee-16-2005/documentos/22ti.html.

Juárez N. S. \& Ehrenzweig S. Y. (2006). Imagen corporal, conductas y trastornos alimentarios en alumnos de un bachiller privado en Xalapa, Veracruz. Tesis de licenciatura en Psicología. Universidad Veracruzana, México

Martínez, J. M. (2001). La importancia de la autoestima en los adolescentes y su relación con el éxito académico.[En línea] Consultado 11/10/05 http://www.universidadabierta.edu.mx/Bliblio/M/Martinez\%20 Melitona-Autoestima.html

Medina, M, Ma. E., Borges, G., Lara, M. C., Benjet, C., Blanco, J. J., Fleiz, B. C., Villatoro, U. J., Rojas, G. E., Zambrano, R. J., Casanova, R. L. \& Aguilar G. S. (2003). Prevalencia de Trastornos Mentales y uso de servicios: Resultados de la encuesta nacional de epidemiología psiquiátrica en México. Salud Mental México, 26(4), 1-16.

Ministerio de Sanidad y Consumo, Servicio Aragonés de salud. (2000). Protocolo de Atención a pacientes con TCA (Anexos I y II), Dirección de atención primaria Hospital Clínico Universitario "Lozano Blesa" sector Zaragoza III.

Montero, P., Morales, E. M. \& Carbajal, A. (2004). Valoración de la percepción de la imagen corporal mediante modelos anatómicos. Antropología, 8, 107-116. www.didac.ehu.es/antropo

Moral de la, R. J. (2002). Los trastornos de la conducta alimentaria, un complejo fenómeno biopsicosocial. Revista de la Facultad de Psicología, 3(3), 1-16.

National Eating Disorders Association (NEDA, 2002). Statistics: eating disorders and their precursors. Vol. 1. Seattle: NEDA,

Organización Mundial de la Salud y Organización Panamericana de la Salud. (1986). Manual de crecimiento y desarrollo del niño. Washington: OPS

Ortuño, B. (1978). Autoestima. En: Martínez J., M. (2001) La importancia de la autoestima en los adolescentes y su relación con el éxito académico. [En línea] Consultado 11/10/05 http://www.universidadabierta. edu.mx/Bliblio/M/Martinez\%20Melitona-Autoestima.html

Quintero, P. E., Pérez, M, A. C., Montiel, N. C., Pirela, D., Acosta, M. F. \& Pineda, N. (2003). Trastornos de la conducta alimentaria. Prevalencia y características clínicas en adolescentes de la ciudad de Maracaibo, Estado Zulia, Venezuela. Revista Investigación Clínica, 44(3), 179-193 [En línea] http://www.scielo.org.ve/scielo.php?

Rosen, J. C. \& Ramírez, E. (1998). A comparison of eating disorders and body dysmorphic disorder o body image and psychological adjustment. Journal of Psychosomatic Res., 44(3-4), 441-449

Ruíz, M. A. O., Vázquez, A. R., Mateo, G. C., Galdós, C. J., Álvarez, R. G. \& Mancilla, D. J. M. (2004) Presencia de trastornos alimentarios y su sintomatología en relación con influencias culturales e insatisfacción corporal entre estudiantes hombres y mujeres. Revista Psicología, 1(1). [En línea] Consultado el 16/IV/08 http://digitalia.uvmnet.edu/ revistadyn/app/articulo/ArticuloDyn.aspx?id=119

Sánchez, V. A., Madrigal, H., Martinez, G. M. A., Kearney, J., Gibney, M. J., Irala, J. \& Martinez, A. (2001). Perception of body image as 
indicator of weight status in the European Union. Journal of Human Nutritional. Diet, 14(2), 93-102

Schvili-Mann, S. \& Dayán-Nahmad, A. (2000), Trastornos de la alimentación: la anorexia nerviosa. Medicina Interna, 16(2), 90-7

Sepúlveda, A. R., Botella, J. \& León J. A. (2001). La alteración de la imagen corporal en los trastornos de la alimentación: Un meta-análisis. Psicothema, 13(1), 7-16

Taylor, S. E. (2007). Conductas que mejoran la salud. En: Taylor, S. E (Ed). Psicología de la Salud. Traducción: Hano, R. M. C. \& Sánchez, P. G. Pp. 16-39. Sexta edición. McGraw-Hill, México.

Toro, J. (1996). El cuerpo como delito. Anorexia, bulimia, cultura y sociedad. Barcelona: Editorial Ariel.
Unikel, S. C. (2000), Conductas alimentarias de riesgo en adolescentes mexicanos. Datos en población estudiantil del DF, México. Revista de Investigación Científica, 52(2), 140-147

Unikel, S.C., Saucedo, V., Villatoro, F. \& Fleiz, V. (2002). Conductas alimentarias de riesgo y distribución del índice de masa corporal en estudiantes de 13 a 18 años. Salud Mental, 25(2), 50-51.

Vázquez, R., Mancilla, J. M., Mateo, C., López, X., Álvarez, G. \& Ruiz, A. (2005). Trastornos del comportamiento alimentario y factores de riesgo de una muestra incidental de jóvenes mexicanos. Revista Mexicana de Psicología, 22(1), 53-63 
